\title{
Auricular acupressure for hot flashes in patients with prostate cancer: protocol for a pilot randomized controlled trial
}

Jianfu Zhou

The Second Affliated Hospital of Guangzhou University of Chinese Medicine

Rongwu Lin

Guangzhou University of Chinese Medicine

Xuehua Liu

Guangzhou University of Chinese Medicine

Liguo Lv

Guangzhou University of Chinese Medicine

Shusheng Wang

Guangzhou University of Chinese Medicine

\section{Zhiqiang Chen}

Guangzhou University of Chinese Medicine

Zhichao Wang ( $13602495696 @ 163 . c 0 m$ )

Guangzhou University of Chinese Medicine

Songtao Xiang ( $\nabla$ tonyxst@gzucm.edu.cn )

The Second Affliated Hospital of Guangzhou University of Chinese Medicine https://orcid.org/00000001-5574-3432

\section{Study protocol}

Keywords: Auricular acupressure, Hot flashes, Prostate cancer, Hormonotherapy, Randomized controlled trial

Posted Date: July 28th, 2020

DOI: https://doi.org/10.21203/rs.3.rs-27959/v1

License: (c) (1) This work is licensed under a Creative Commons Attribution 4.0 International License.

Read Full License 
7 Authors' affiliations: ${ }^{1}$ Department of Urology, The Second Affiliated Hospital of 8 Guangzhou University of Chinese Medicine, Guangzhou 510370, China; ${ }^{2}$ The Second 9 Clinical College, Guangzhou University of Chinese Medicine, Guangzhou 510145, China

10 Running title: Auricular acupressure for hot flashes in prostate cancer

12

\section{Auricular acupressure for hot flashes in patients with prostate cancer:} protocol for a pilot randomized controlled trial

$$
\begin{gathered}
\text { Jianfu Zhou }{ }^{1,2, \#} \text {, Rongwu Lin }{ }^{2, \#}, \text { Xuehua Liu }{ }^{1} \text {, Liguo Lv¹, Shusheng Wang }{ }^{1} \text {, } \\
\text { Zhiqiang Chen }{ }^{1,} \text { Zhichao Wang }{ }^{1, *} \text { and Songtao Xiang }{ }^{1, *}
\end{gathered}
$$

6

Keywords: Auricular acupressure, Hot flashes, Prostate cancer, Hormonotherapy,

Randomized controlled trial

${ }^{\#}$ Jianfu Zhou and "Rongwu Lin contributed equally to this article.

\section{*Correspondence:}

Zhichao Wang and Songtao Xiang, Department of Urology, The Second Affiliated Hospital of Guangzhou University of Chinese Medicine, Fangcun Branch, 36 Chong'an Street, North Dongjiao Road, Guangzhou 510370, China; Phone: +86 (020) 8149 9399-8333; Fax: +86 (020) 81499866 ;

E-mails: 13602495696@163.com (Z.W.); tonyxst@gzucm.edu.cn (S.X.) 


\section{Abstract}

27 Background: Hot flashes, characterized by intense heat sensation and diaphoresis, are common side effects resulted from hormonotherapy in patients with prostate cancer. Cumulated studies have revealed beneficial role of acupuncture as complementary and alternative recipe for the management of hot flashes. However, little is known about the auricular acupressure (AA), a micro-acupuncture technique whose therapeutic purpose is similar with conventional acupuncture. Therefore, this current study aims to explore the effects and determine the feasibility of AA for hot flashes in patients with prostate cancer.

Methods/Design: This proposed pilot study is a two-arm parallel, single-blinded, randomized sham-controlled trial. A total of 72 participants of prostate cancer suffered with hot flashes will be recruited and randomly allocated into two groups in a 1:1 ratio. Equal randomization is conducted using a computer-generated random allocation sequence. Sheng Zhi Qi (TF2), Nei Fen Mi (CO18), Shen Men (TF4), Shen (CO10) and Pi Zhi Xia (AT4) are selected as experimental acupressure points, and five helix points (HX 8-12) are used as sham control acupressure points. Participants in the experimental group and control group will receive AA and sham-AA treatment, respectively. The duration of the treatment is 6 weeks with two sessions per week, and the follow-up period is 12 weeks. The primary outcome is Hot Flash Score (HFS). The secondary outcomes include Quality of Life (QoL), Pittsburgh Sleep Quality Index (PSQI) and Hamilton Anxiety Scale (HAS). All outcomes measurement will be conducted before and through treatment period as well as follow-up period. Safety assessment will be carried out through treatment and follow-up period.

47 Discussion: This pilot study will for the first time advance our knowledge on feasibility of AA in alleviating hot flashes in patients of prostate cancer and provide preliminary evidence 49 for a further full-scale trial. 
October 2019.

\section{Background}

Prostate cancer is one of the most common cancer types and the second leading cause of cancer related death in men worldwide [1]. Hormonotherapy, carried out by surgical or medical castration combined with antiandrogen, has been standard modality for the treatment of locally advanced and metastatic prostate cancer [2,3]. Although this therapy can be quite efficacious, it, concomitantly, is associated with a range of side effects [4-6]. One of the wellknown and uncomfortable side effects is the experience of hot flashes, which occurs in 50\% to $80 \%$ of patients with advanced prostate cancer receiving hormonotherapy [7-9]. Hot flashes, characterized by subjective sensations of heat or sweating, are often associated with psychosomatic disorders and adversely affect the quality of life [10,11]. Given that the hormonotherapy is usually lifelong for patients of advanced prostate cancer, manipulation of potential hot flashes accompanied is urgently needed.

The pathophysiology of hot flashes is quite complex and has not been fully understood, thus management of these symptoms remains challenging [12-14]. A variety of medical options have been evaluated for alleviating hot flushes, including hormonal replacement therapies (e.g., estrogen, progesterone analogs and cyproterone acetate), non-hormonal drug treatments such as gabapentin, clonidine and selective serotonin reuptake inhibitors (e.g., venlafaxine, paroxetine) [15-17]. Most of these treatments have been assessed mainly in postmenopausal women and particularly breast cancer patients undergoing hormonotherapy [18]. There have been far fewer studies in men for patients of prostate cancer treated with hormonotherapy. While moderately effective, both hormonal and non-hormonal therapies are costly and have been associated with a series of unfavorable complications including nausea, 
dizziness, and anxiety [15-17]. Thus, more effective treatments with fewer side effects remain to be explored for hot flashes.

Complementary and alternative therapies have been used successfully to treat a variety of health problems for few or no side effects. Previous studies demonstrated that traditional Chinese medicine formula [19], medicinal herb [20], as well as phytoestrogens [21] were effective in treating hot flashes. Acupuncture, a popular and recognized complementary and alternative therapy, has also shown beneficial effects for the management of hot flashes in post-menopausal women with or without breast cancer [22], and in men with prostate cancer [23]. Auricular acupressure (AA), a micro-acupuncture technique whose therapeutic purpose is similar with conventional acupuncture, has been investigated in improving sleep quality, relieving menopausal anxiety and hot flashes [24-26]. Harding et al. reported that auricular acupuncture might be a useful treatment for hot flashes in men with prostate cancer upon medicinal castration treatment [27]. However, little is known about AA for the treatment of hot flashes in patients with prostate cancer.

In the current study, we aim to perform a pilot, randomized and controlled trial (RCT) to determine whether AA is effective and safe as compared to a sham-AA control for the management of hot flashes in patients with prostate cancer. The results of this trial will form an informative basis of feasibility and provide preliminary evidence guiding a further fullscale trial.

\section{Methods/Design}

\section{Design}

This pilot study is a prospective, two-arm parallel, single-blinded, randomized, shamcontrolled clinical trial, and the protocol is presented according to items recommendation of 
100 AA experimental group and the sham-AA control group in a 1:1 allocation ratio. Equal

101 randomization will be conducted using a computer-generated random allocation sequence.

102 All participants will be required to sign the informed consent before proceeding into the trial.

103 The schematic flow chart of the study process is shown in Figure 1, and the participant

104 timeline with events schedule is provided in Figure 2.

105

106 Participants

107 Setting and recruitment

108 This trial will be conducted in The Second Affiliated Hospital of Guangzhou University

109 of Chinese Medicine. Participants of the study is recruited through the outpatient clinic,

110 hospital-based advertising, and posters. The trial protocol is in accordance with the principles

111 of the Declaration of Helsinki and has been approved by Institutional Ethics Committee

112 review board (approval number: B2017-119-01) of Guangdong Provincial Hospital of

113 Chinese Medicine (The Second Affiliated Hospital of Guangzhou University of Chinese

114 Medicine). Written informed consent will be obtained from each participant. This trial was

115 registered at the Chinese Clinical Trial Registry (ChiCTR1900026694).

116

117 Eligibility criteria

118 Inclusion criteria

119 1) Histologic diagnosis of prostate cancer with a history of hormonotherapy use;

120 2) Experienced hot flashes at least a month before study entry;

121 3) Men 18 years or older with expectative life expectancy more than three months;

122 4) Willingness to participate in the study and sign informed consent;

123 5) Karnofsky Performance Status $\geq 60$. 
126 1) Patients with a primary malignancy other than prostate cancer;

127 2) Under treatment for hot flashes control by using gabapentin, venlafaxine, etc.;

128 3) Unable to receive AA treatment and known allergy constitution;

129 4) Those who with severe heart, brain, kidney, liver, infectious or mental disease;

130 5) Difficulties in cooperating with the researchers and filling out the study documents.

\section{Withdrawal criteria}

Participants will be withdrawn from this study in the following situations:

1) When a participant requests to withdraw from the study, for any reason, at any time;

2) When worsening disease or severe adverse events or reactions take place.

\section{Randomization, allocation concealment and blinding}

Eligible participants will be randomly assigned to either an experimental group receiving AA intervention, or a control group receiving sham-AA intervention in a 1:1 ratio.

141 Equal randomization will be carried out using a computer-created random allocation

142 sequence through the method of stratified block randomization by the SAS 9.2 software (SAS

143 Institute Inc., Cary, NC, USA). The research coordinator will access for the treatment

144 allocation information for each eligible participant through an online system, which was

145 developed by the Key Unit of Methodology in Clinical Research, The Second Affiliated

146 Hospital of Guangzhou University of Chinese Medicine. The other personnel, including

147 clinical physicians, AA practitioners, and assessors, will not be authorized to apply for 148 randomization numbers. Treatment allocations will be blinded to participants, assessors and 149 statisticians, and will not be revealed until the trial is completed. In order to avoid the 
150 influence of the Rosenthal and Hawthorne effects, the AA practitioners will be restricted to 151 communicate with the participants $[28,29]$.

\section{Interventions}

This trial includes a 6-week treatment period with two sessions per week, and a 12-week post-treatment period with follow-up of every 3 weeks. AA and sham-AA will be conducted in participants of experimental group and control group, respectively. The auricular points in both groups are illustrated in Figure 3, and the locations of these points are listed in Table 1, which are both in accordance with the National Standards of the Nomenclature and Location of Auricular Acupoints published in China [30].

160 AA manipulation will be delivered through pressure stimulation on auricular points using Semen Vaccaria seeds (Wang-Bu-Liu-Xing). Briefly, after sterilization with 75\% alcohol, a $1.0 \mathrm{~cm} \times 1.0 \mathrm{~cm}$ adhesive plaster with one bead imbedded will be attached and fixed on the specific auricular points. The patients will be asked to press the auricular points by

164 themselves 4-6 times a day for a 3-minutes duration each time. The AA manipulation will be conducted alternatively between the two ears every 2 days. The plaster with seeds will be exchanged for a fresh set once a week

\section{Experimental group}

169 Acup. 1. Sheng Zhi Qi (Internal Genitals, TF2)

Acup. 2. Nei Fen Mi (Endocrine, CO18)

Acup. 3. Shen Men (Spiritual Gate, TF4) 
Participants in the control group receive sham-AA treatment on five helix points (HX 8-

177 12), which are clearly remote from the inner ear area. These auricular points lack evidence for 178 hot flashes control. Previous studies indicated that these kinds of auricular points had minor 179 effects on subjective symptoms regulation including improving sleep quality and relieving anxiety situation, which could serve as placebo effect [31,32].

181

\section{Outcome measures}

\section{Primary outcome}

184 To quantify the hot flashes, the eligible subjects will be required to document the frequency and severity of hot flashes, by using daily hot flash diary described previously [33]. Briefly, each participant will record how many hot flashes he experiences each day, meanwhile how many are mild, moderate, severe, or very severe. The Hot Flashes Score (HFS) of each day can be calculated into 1,2,3, or 4, respectively, by multiplying the number of hot flashes recorded as mild, moderate, severe, or very severe with adding distinct values to obtain a composite score.

\section{Secondary outcomes}

Secondary outcome measures include changes in Quality of Life (QoL), Pittsburgh

194 Sleep Quality Index (PSQI) and Hamilton Anxiety Scale (HAS) [34]. All the primary and

195 secondary outcome measures will be evaluated at baseline visit and repeated among treatment 196 period as well as follow-up period.

197

\section{Safety assessment}


199 All participants will undergo laboratory tests, including evaluation of hematologic and

200 urinary routine tests, blood biochemical tests (renal and hepatic function) and

201 electrocardiograph, before the start of treatment and after 6 weeks of treatment. The

202 participants will be asked to report information about potential adverse events (AEs) such as

203 local skin irritation and discomfort, light tenderness or pain, and dizziness during AA

204 treatment [35]. In case of severe AEs, AA treatment will be discontinued immediately. All

205 AEs will be fully recorded on the AEs pages of the case report forms (CRFs). The researcher

206 will confirm the occurrence of AEs and record all details including the occurrence date,

207 duration, degree, and causal relationship with the treatment. Emergency medical assistance

208 will be provided if any serious AEs occurs, and all details will be noted.

210 Sample size estimation

211 This study aims to evaluate clinical trial feasibility and to investigate basic information

212 about the efficacy and safety of AA for the treatment of hot flashes in patients with prostate 213 cancer, rather than hypothesis testing. Therefore, the sample size was decided based on a 214 rationale for feasibility, which were unable to calculate the statistical power formally. In 215 reference of a previous similar study of acupuncture for the treatment of hot flashes in breast

216 cancer patients [36], the present research thus incorporated a sample size of 30 for each group.

217 Considering an estimated $15 \%$ dropout rate, a required sample size in each group was 218 estimated to 36 . In total, 72 participants will be enrolled in this trial.

Data collection and management

All data will be recorded on the hard copy of CRFs. Data regarding the demographic characteristics and the baseline assessment will be collected by the screeners when the participants are recruited. Outcome measurements will be performed by assessors through the 
treatment period and the follow-up period. Data of prescription and any AEs reported by participants will be collected by clinicians. A research coordinator will perform quality control of data collection and be responsible for data access.

Monitoring

The Key Unit of Methodology in Clinical Research of Guangdong Provincial Hospital of Chinese Medicine (The Second Affiliated Hospital of Guangzhou University of Chinese Medicine) is the Monitoring Committee for Medical Data in this trial. All data will be recorded by designated outcome assessors on the hard copy of CRFs, and double-entered into the electronic CRFs, which are established and monitored by the Key Unit of Methodology in Clinical Research. Monitors will audit the data every three months. AA practitioners and statisticians will have no access to these data during the evaluation process.

\section{Statistical methods}

All data will be presented as means and standard deviations or number (percentage), and all analyses will be based on the intention-to-treat principle. For the description of baseline characteristics, the mean with standard deviation or range with the minimum and maximum values for continuous data and frequency with percentage for dichotomous data will be reported. Homogeneity between the two groups in terms of baseline characteristics will be tested using the two-sample $t$ test for continuous data and the chi square $\left(\chi^{2}\right)$ test for

244 dichotomous data. Analysis of covariance (ANCOVA) or logistic regression will be used for analysis and adjustment of baseline characteristic that differ significantly between the two groups.

\section{Discussion}


As far as we know, this will be the first study to evaluate the effectiveness and safety of

250 AA for the treatment of hot flashes in patients of prostate cancer receiving hormonotherapy.

251 Although AA has been practiced as a complementary treatment for anxiety, insomnia, peri252 and early postmenopausal symptoms, there is still an important gap of AA for hot flashes 253 control in patients of prostate cancer. The results of this study are expected to offer preliminary evidence regarding for AA in treating hot flashes in patients of prostate cancer.

Pilot studies, also known as feasibility studies, are prospective comparative trials commonly designed for providing preliminary evidence towards the clinical efficacy of a specific treatment or intervention [37]. A standardized protocol will be used to ensure the reproducibility of the pilot randomized controlled trial. This study has been designed carefully in accordance with the Consolidated Standards of Reporting Trials (CONSORT) Statement of RCTs and presented the protocol according to the Standard Protocol Items: Recommendations for Interventional Trials (SPIRIT) Statement (Additional file 1). Methodological benchmarks such as randomization, allocation concealment and blinding has been robustly met in the protocol. The chosen primary outcome is change in frequency and severity of hot flashes by HFS, which is known to be valid to treatment effects [33].

There are several limitations in this pilot study. First of all, this pilot trial will include a small sample size of participants and hypothesis testing will not be involved. For this reason,

267 the results of this trial are not capable to generate adequate data for assessing the efficacy and 268 safety of AA for the treatment of hot flashes. In addition, the treatment of hot flashes with 269 AA is not based on syndrome differentiation, which is the major concern in traditional 270 Chinese medicine. The selection of auricular points is standardized and put on every 271 participant, this may be convenient for better use of the treatment over different individuals. 272 Moreover, AA practitioner will not be blinded because of the nature of the intervention. In 273 order to avoid the influence of the Hawthorne and Rosenthal effects, the AA practitioners 
will be restricted to communicate with the participants and will not be involved in assessing outcomes or the data analysis.

To summarize, this study protocol describes the first randomized, sham-controlled trial

277 for evaluating feasibility of AA in alleviating hot flashes in patients of prostate cancer. Our

278 results will inform men suffering with hot flashes and both conventional and Traditional

279 Chinese Medicine healthcare professionals on the potential role, if any, of AA in the treatment of hot flashes. The findings of the study will provide evidence for a further fullscale RCT trial.

Trial status

This trial is currently in ongoing phase. The protocol version number is PRO1.1. Patient recruitment began in January 2020 and is expected to be completed by December 2022 .

\section{Additional files}

Additional file 1: SPIRIT 2013 Checklist: recommended items to address in a clinical trial protocol and related documents.

\section{Abbreviations}

AA: auricular acupressure; Sham-AA: Sham auricular acupressure; RCT: Randomized controlled trial; HFS: Hot Flashes Score; QoL: Quality of Life; PSQI: Pittsburgh Sleep

294 Quality Index; HAS: Hamilton Anxiety Scale; AEs: Adverse events; CRFs: Case report forms; CONSORT: Consolidated Standards of Reporting Trials; SPIRIT: Standard Protocol Items: Recommendations for Interventional Trials. 
JZ designed the trial and drafted the manuscript. RL assisted and prepared documents

300 for ethics review. XL, LL coordinated and provided important suggestions. SW and ZC

301 supervised the work with critical revision of the manuscript. JZ, ZW and SX obtained

302 funding for supporting this trial. ZW and SX contributed to the conception and finalized the

303 manuscript. All authors read and approved the final manuscript

Acknowledgements

306

Many thanks to Cecilia Stålsby Lundborg from Department of Public Health Sciences,

Global Health-Health Systems and Policy, Karolinska Institutet for her training course on methodology of clinical research. The authors are also grateful to Wenwei Ouyang from the

Key Unit of Methodology in Clinical Research of Guangdong Provincial Hospital of Chinese

Medicine for his informative suggestion on sample size estimation.

\section{Funding}

313 This work was supported in part by the Scientific Research Project Funded by

314 Traditional Chinese Medicine Bureau of Guangdong Province (No. 20172068), Natural

315 Science Foundation of Guangdong Province (No. 2017A030310501), Medical Science and

316 Technology Research Fund of Guangdong Province (No. A2020239), Guangdong-Hong

317 Kong collaborative innovation projects from Guangdong Provincial Hospital of Chinese

318 Medicine (No. YN2018HK02) and Department of Science and Technology of Guangdong

319 Province (No. 2017A050506042). JZ was awarded by the Eternal Flame Scholar Programme

320 of Guangzhou University of Chinese Medicine. The funders had no role in the study design

321 or decision to submit the manuscript for publication.

323 Availability of data and materials 
Not applicable.

\section{Ethics approval and consent to participate}

This study protocol had been reviewed and approved by Institutional Ethics Committee review board (approval number: B2017-119-01) of Guangdong Provincial Hospital of

329 Chinese Medicine (The Second Affiliated Hospital of Guangzhou University of Chinese 330 Medicine). Any important modifications will be immediately communicated to the 331 Institutional Ethics Committee and Data Monitoring Committee for amendments. The 332 modifications will also be updated on the Chinese Clinical Trial Registry. Written informed 333 consent will be obtained from all eligible participants before the allocation.

\section{Consent for publication}

Not applicable.

\section{Conflict of interest}

The authors declared that no conflict of interest exists.

\section{Author details}

${ }^{1}$ Department of Urology, The Second Affiliated Hospital of Guangzhou University of

343 Chinese Medicine, Guangzhou 510370, China; ${ }^{2}$ The Second Clinical College, Guangzhou

344 University of Chinese Medicine, Guangzhou 510145, China

\section{References}

347 1. Bray F, Ferlay J, Soerjomataram I, et al. Global cancer statistics 2018: GLOBOCAN 348 estimates of incidence and mortality worldwide for 36 cancers in 185 countries. CA 349 Cancer J Clin. 2018;68(6):394-424. 
2. Teo MY, Rathkopf DE, Kantoff P. Treatment of advanced prostate cancer. Annu Rev Med. 2019;70:479-99.

3. Zhou J, Wang Y, Xiang S, et al. Towards understanding androgen receptor-independent prostate cancer: an evolving paradigm. Transl Cancer Res. 2020;9(2):415-7.

4. Ahmadi H, Daneshmand S. Androgen deprivation therapy: evidence-based management of side effects. BJU Int. 2013;111(4):543-8.

5. Allan CA, Collins VR, Frydenberg M, et al. Androgen deprivation therapy complications. Endocr Relat Cancer. 2014;21(4):T119-29.

6. Nguyen PL, Alibhai SM, Basaria S, et al. Adverse effects of androgen deprivation therapy and strategies to mitigate them. Eur Urol. 2015;67(5):825-36.

7. Karling P, Hammar M, Varenhorst E. Prevalence and duration of hot flushes after surgical or medical castration in men with prostatic carcinoma. J Urol. 1994;152(4):1170-1173.

8. Schow DA, Renfer LG, Rozanski TA et al. Prevalence of hot flushes during and after neoadjuvant hormonal therapy for localized prostate cancer. South Med J. 1998;91(9):855-7.

9. Sakai H, Igawa T, Tsurusaki T, et al. Hot flashes during androgen deprivation therapy with luteinizing hormone-releasing hormone agonist combined with steroidal or nonsteroidal antiandrogen for prostate cancer. Urology. 2009;73(3): 635-40.

10. Gonzalez BD, Jim HSL, Donovan KA, et al. Course and moderators of hot flash interference during androgen deprivation therapy for prostate cancer: A matched comparison. J Urol. 2015;194(3):690-5.

11. Nishiyama T, Kanazawa S, Watanabe R, et al. Influence of hot flashes on quality of life in patients with prostate cancer treated with androgen deprivation therapy. Int J Urol. 2004;11(9):735-41.

12. Stearns V, Ullmer L, López JF, et al. Hot flushes. Lancet. 2002;360(9348):1851-61.

13. Kouriefs C, Georgiou M, Ravi R. Hot flushes and prostate cancer: pathogenesis and treatment. BJU Int. 2002;89:379-83.

14. Freedland SJ, Eastham J, Shore N. Androgen deprivation therapy and estrogen deficiency induced adverse effects in the treatment of prostate cancer. Prostate Cancer Prostatic Dis. 2009;12(4):333-8.

15. Stearns V. Clinical update: new treatments for hot flushes. Lancet. 2007;369 (9579):2062-4.

16. Morrow PK, Mattair DN, Hortobagyi GN. Hot flashes: a review of pathophysiology and treatment modalities. Oncologist. 2011;16(11):1658-64. 
17. Jones JM, Kohli M, Loprinzi CL. Androgen deprivation therapy-associated vasomotor symptoms. Asian J Androl. 2012;14(2):193-7.

18. Wiśniewska I, Jochymek B, Lenart-Lipińska M, et al. The pharmacological and hormonal therapy of hot flushes in breast cancer survivors. Breast Cancer. 2016; 23(2):178-82.

19. Xue D, Sun H, Li PP. Long-term chinese herbs decoction administration for management of hot flashes associated with endocrine therapy in breast cancer patients. Chin J Cancer Res. 2011;23(1):74-8.

20. Vandecasteele K, Ost P, Oosterlinck W, et al. Evaluation of the efficacy and safety of Salvia officinalis in controlling hot flashes in prostate cancer patients treated with androgen deprivation. Phytother Res. 2012;26(2):208-13.

21. Vitolins MZ, Griffin L, Tomlinson WV, et al. Randomized trial to assess the impact of venlafaxine and soy protein on hot flashes and quality of life in men with prostate cancer. J Clin Oncol. 2013;31(32):4092-8.

22. Nir Y, Huang MI, Schnyer R, et al. Acupuncture for postmenopausal hot flashes. Maturitas. 2007;56(4):383-95.

23. Lee MS, Kim KH, Shin BC, et al. Acupuncture for treating hot flushes in men with prostate cancer: a systematic review. Support Care Cancer. 2009;17(7):763-70.

24. Zhou J, Qu F, Sang X, et al. Acupuncture and auricular acupressure in relieving menopausal hot flashes of bilaterally ovariectomized chinese women: a randomized controlled trial. Evid Based Complement Alternat Med. 2011:713274.

25. Kao CL, Chen CH, Lin WY, et al. Effect of auricular acupressure on peri- and early postmenopausal women with anxiety: a double-blinded, randomized, and controlled pilot study. Evid Based Complement Alternat Med. 2012:567639.

26. Wu Y, Zou C, Liu X, et al. Auricular acupressure helps improve sleep quality for severe insomnia in maintenance hemodialysis patients: a pilot study. $\mathrm{J}$ Altern Complement Med. 2014;20(5):356-63.

27. Harding C, Harris A, Chadwick D. Auricular acupuncture: a novel treatment for vasomotor symptoms associated with luteinizing-hormone releasing hormone agonist treatment for prostate cancer. BJU Int. 2009;103(2):186-90.

28. Mitchell TR, Daniels D. Motivation. In: Borman WC, Ilgen DR, Klimoski RJ, editors. Handbook of psychology. Hoboken: Wiley; 2003. p. 229.

29. McCarney R, Warner J, Iliffe S, van Haselen R, Griffin M, Fisher P. The Hawthorne Effect: a randomised, controlled trial. BMC Med Res Methodol. 2007;7:30. 
30. Zhou LQ, Zhao BX. Nomenclature and location of auricular points. Beijing, China: Standard Publishing House; 2008

31. Zou C, Yang L, Wu Y, et al. Auricular acupressure on specific points for hemodialysis patients with insomnia: a pilot randomized controlled trial. PLoS One. 2015;10(4):e0122724.

32. Wu Y, Yang L, Li L, et al. Auricular acupressure for insomnia in hemodialysis patients: study protocol for a randomized controlled trial. Trials. 2018;19(1):171.

33. Mao JJ, Bowman MA, Xie SX, et al. Electroacupuncture versus gabapentin for hot flashes among breast cancer survivors: a randomized placebo-controlled trial. J Clin Oncol. 2015;33(31):3615-20.

34. Milajerdi A, Jazayeri S, Shirzadi E, et al. The effects of alcoholic extract of saffron (Crocus satious L.) on mild to moderate comorbid depression-anxiety, sleep quality, and life satisfaction in type 2 diabetes mellitus: A double-blind, randomized and placebocontrolled clinical trial. Complement Ther Med. 2018; 41:196-202.

35. Li Q, Zhang BP, Huang SG, et al. Efficacy and indication optimization of Chinese medicine (Tiao-Chang Ke-Min granules) for diarrhea-predominant irritable bowel syndrome: study protocol for a randomized controlled trial. Trials. 2018;19(1):367.

36. Deng G, Vickers A, Yeung S, et al. Randomized, controlled trial of acupuncture for the treatment of hot flashes in breast cancer patients. J Clin Oncol. 2007;25(35):5584-90.

37. Leon AC, Davis LL, Kraemer HC. The role and interpretation of pilot studies in clinical research. J Psychiatr Res. 2011;45(5):626-9.

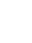

2

3




\section{Figure Legends}

453

$454 \quad$ Figure 1 Schematic flow chart of the study process.

455

456 Figure 2 Participant timeline with events schedule. AA: auricular acupressure; Sham-AA: 457 Sham auricular acupressure; HFS: Hot Flashes Score; QoL: Quality of Life; PSQI: Pittsburgh 458 Sleep Quality Index; HAS: Hamilton Anxiety Scale.

459

460 Figure 3 Auricular acupoints. Red circle indicates auricular acupoints used in experimental 461 group receiving auricular acupressure (AA), while green circle regards auricular acupoints 462 selected in control group undergoing sham-auricular acupressure (sham-AA).

463

464 Table 1 Locations of auricular acupoints used in the trial. 
Figures

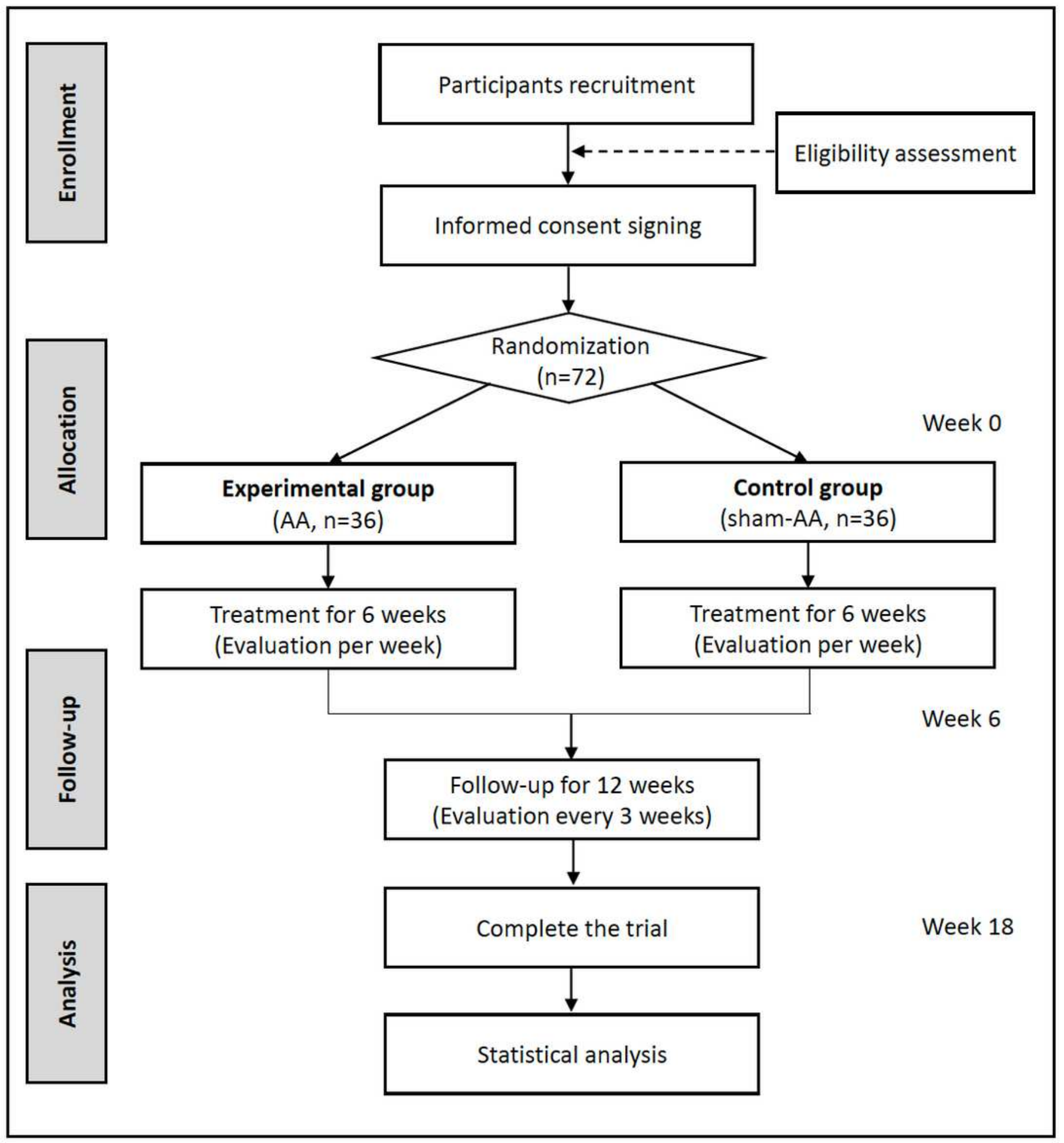

Figure 1

Schematic flow chart of the study process. 


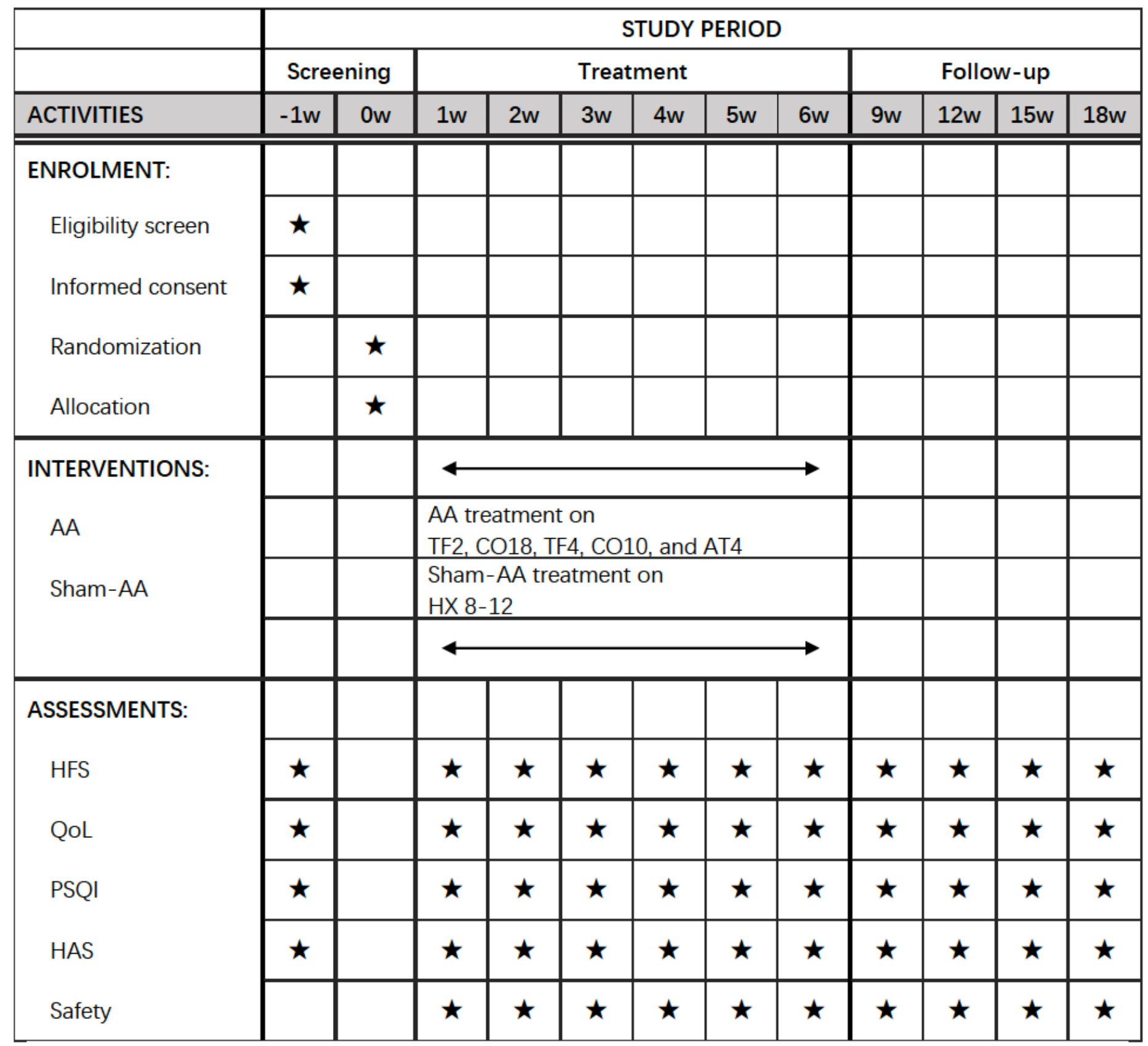

Figure 2

Participant timeline with events schedule. AA: auricular acupressure; Sham-AA: Sham auricular acupressure; HFS: Hot Flashes Score; QoL: Quality of Life; PSQI: Pittsburgh Sleep Quality Index; HAS: Hamilton Anxiety Scale. 


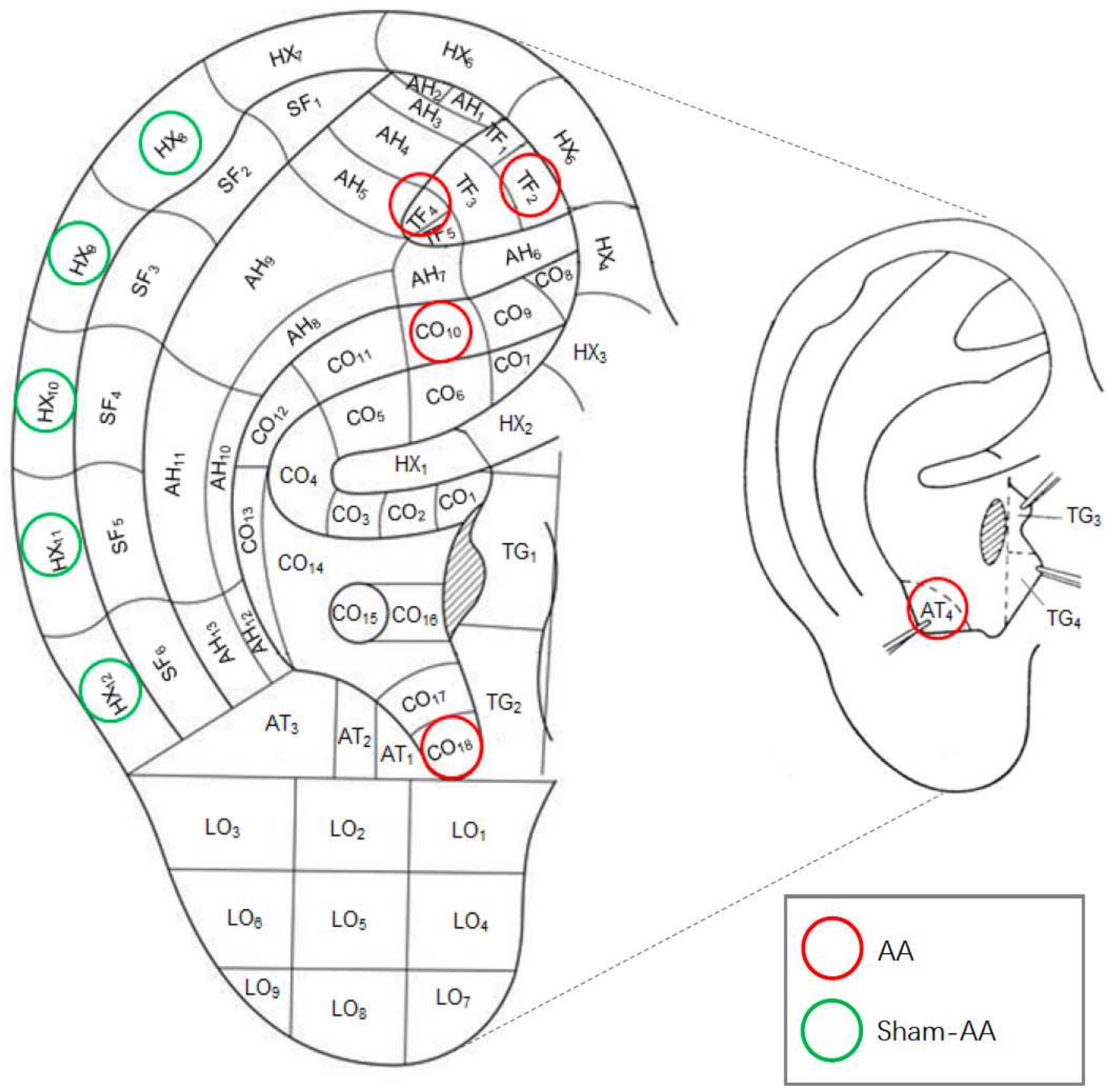

Figure 3

Auricular acupoints. Red circle indicates auricular acupoints used in experimental group receiving auricular acupressure (AA), while green circle regards auricular acupoints selected in control group undergoing sham-auricular acupressure (sham-AA).

\section{Supplementary Files}


This is a list of supplementary files associated with this preprint. Click to download.

- SPIRIT2013Checklist.docx 